\title{
SISTEMA AMBULATORIO DE MONITORIZACIÓN DE CRISIS EPILÉPTICAS
}

\author{
D. Zambrana-Vinaroz, J.M. Sabater-Navarro \\ Universidad Miguel Hernández, Elche-España, dzambrana@umh.es
}

\begin{abstract}
Resumen
La epilepsia es una enfermedad crónica con un importante impacto social. Los afectados y sus familiares suelen vivir condicionados por la posibilidad de una crisis epiléptica y sus posibles consecuencias como accidentes, lesiones $e$ incluso la muerte súbita inexplicable (SUDEP). Por ello, en este artículo se propone el diseño de un dispositivo electrónico y portátil que integra y guarda la información recogida por diferentes sensores (EEG, ECG y PPG) con el fin de calcular ciertos parámetros que aportan información acerca del sistema nervioso y cardiovascular. Además, dicho dispositivo puede ser utilizado para estudiar de manera ambulatoria las crisis epilépticas y servir como instrumento de apoyo a la hora de tomar decisiones clínicas.
\end{abstract}

Palabras clave: Epilepsia, monitorización portátil, ECG, EEG, PPG, PTT.

\section{INTRODUCCIÓN}

La epilepsia es una enfermedad crónica de enorme impacto sociosanitario. La tasa de prevalencia global en España es de 14,87 casos por 1.000 habitantes [9] y su incidencia global en el mundo oscila alrededor de los 50 casos por 100.000 habitantes y año [1], [11]. Aunque disponemos de una gran cantidad de fármacos antiepilépticos y de otros tratamientos más selectivos como la cirugía o la estimulación cerebral, un porcentaje considerable de pacientes (alrededor del $30 \%$ ) no están controlados y continúan teniendo crisis epilépticas, por lo que es necesario optimizar la atención de la epilepsia en toda Europa y continuar con los esfuerzos de investigación actuales.

La tasa de mortalidad de los pacientes con epilepsia es de 2 a 3 veces mayor a la de la población general y son relativamente frecuentes los suicidios, los accidentes y la muerte súbita inexplicable [1], [11], [2]. La muerte súbita inexplicada en epilepsia (SUDEP) hace referencia a la muerte inesperada de una persona con epilepsia por lo demás sana, sin que se encuentre una causa clara [6]. Los mecanismos exactos son desconocidos, aunque se considera que una disfunción cardiorrespiratoria provocada por cambios directos en el control autonómico respiratorio y cardiaco (apnea, bradicardia postictal y parada cardiaca) durante o en los momentos inmediatamente posteriores a una crisis generalizada tónico-clónica podría ser la responsable de este fatal desenlace [13].

En este contexto, podemos decir que la falta de previsibilidad de las crisis y sus posibles y graves consecuencias constituye una importante limitación de la independencia de las personas epilépticas y condiciona enormemente su calidad de vida.

Por ello, es necesario llevar a cabo una monitorización ambulatoria de ciertas variables bioeléctricas que permitan llevar a cabo un análisis del estado de salud de las personas [14].

La variabilidad del ritmo cardíaco (HRV heart rate variability) es el fenómeno fisiológico de la variación en el intervalo de tiempo entre cada latido cardíaco, es decir, el tiempo entre un pico $\mathrm{R}$ y el siguiente pico $\mathrm{R}$ de la señal de electrocardiograma (ECG) [15]. Como muchos otros sistemas (sistema renal, sistema digestivo, etc.), el sistema cardiovascular está vinculado con el sistema nervioso central. La fotopletismografia (PPG) [5] es una técnica óptica, no invasiva, que es usada para detectar cambios en el volumen de la sangre en la capa microvascular del tejido. La señal del voltaje de PPG es proporcional a la cantidad de sangre que atraviesa los vasos sanguíneos, por lo que es capaz de detectar la onda de pulso que se transmite por los vasos sanguíneos.

La frecuencia cardíaca está bajo control del sistema nervioso autónomo, que tiene una rama simpática y parasimpática. En una condición de estrés o de enfermedad surge una predominancia del sistema nervioso simpático que hace que el sistema eléctrico del corazón se vuelva inestable. Por lo tanto, es un marcador del estado de activación relativa del eje simpático-parasimpático [12],[4].

El tiempo de tránsito del pulso (PTT) es el tiempo que tarda la forma de onda de la presión del pulso (PP) en 
propagarse a lo largo del árbol arterial. En concreto, el dispositivo que se propone es capaz de medir el tiempo transcurrido desde que se produce la onda $\mathrm{R}$ del electrocardiograma hasta que la onda de pulso captada por el sensor de fotopletismografía (situado en el dedo índice de la mano izquierda) llega a su valor máximo. El parámetro PTT es usado como un indicador de cambio de la presión arterial [3]. Dicho parámetro es inversamente proporcional a la presión sanguínea, debido a que un aumento en la presión arterial provoca que la onda de pulso viaje antes al lugar de la periferia de medida (dedo índice) reduciéndose de esta manera el tiempo de transito de pulso. Sin embargo, cuando la presión arterial disminuye, el tiempo de transito de pulso aumenta.

En todas estas señales descritas anteriormente, la definición está basada en una toma de datos estática, donde no hay movimiento del cuerpo del sujeto, y por lo tanto la relación ruido-señal es la menor posible.

El presente trabajo muestra un sistema concebido para la toma ambulatoria de dichas señales, y que tiene por objeto obtener una información de un nivel de calidad similar al que se obtiene con los sistemas estáticos usados en las unidades de videovigilancia de los hospitales.

\section{METODOLOGÍA}

\subsection{OpenBCI}

El sistema de monitorización de crisis epilépticas se basa en una placa OpenBCI [8]. La placa OpenBCI Cyton (Figura 1) es una interfaz neuronal de 8 canales que se utiliza para medir y registrar la actividad eléctrica producida por el cerebro (EEG) siendo compatibles con los electrodos de EEG estándar. Dicha placa lleva implementada un microcontrolador PIC32MX250F128B (el cual puede ser reprogramado), lo que le proporciona mucha memoria local y velocidades de procesamiento rápidas. Además, la placa contiene:

- Un circuito integrado desarrollado por Texas Instruments para mediciones de biopotencial con 24 bits de resolución (ADS1299).

- Un acelerómetro de 3 ejes (LIS3DH).

- Módulo para guardar los datos en una tarjeta SD.

- Módulo de radio (para conectarse y comunicarse con un dongle conectado a un ordenador o tablet).

Cabe destacar que el microcontrolador trabaja a una tensión de $3,3 \mathrm{~V}$ y se comunica con el resto de integrados de la placa mediante el protocolo de comunicación de SPI. La sincronización y la transmisión de datos se realiza por medio de 4 señales, siendo el master el PIC32 y los esclavos el resto de integrados que contiene (ADS1299, LIS3DH, Tarjeta $\mathrm{SD})$ :

- SCLK (Clock): Es el pulso que marca la sincronización. Con cada pulso de este reloj, se lee o se envía un bit. Dicha señal tiene una frecuencia de $4 \mathrm{MHz}$.

- MOSI (Master Output Slave Input): Salida de datos del Master y entrada de datos al Esclavo.

- MISO (Master Input Slave Output): Salida de datos del Esclavo y entrada al Master.

- SS/Select: Sirve para que el Master seleccione al Esclavo con el que se quiere comunicar.

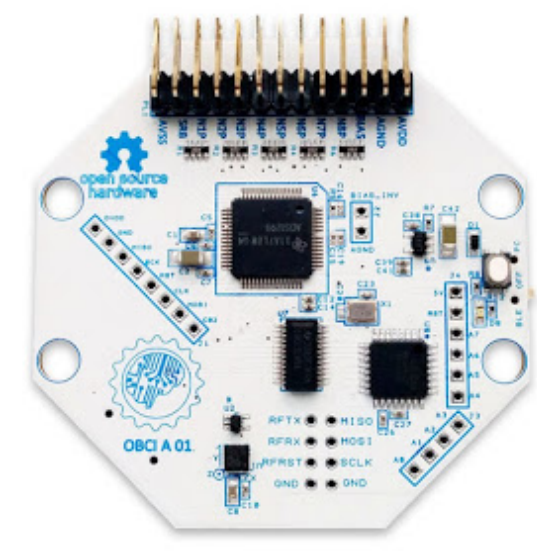

Figura 1: OpenBCI

\subsection{MÓDULO MAX86150}

El módulo de biosensor MAX86150 [7] de Maxim Integrated ofrece mediciones de fotopletismografía (PPG) y electrocardiograma (ECG) en un único paquete integrado con 16 bits de resolución. El módulo MAX86150 combina LEDs internos, fotodetectores y un Front-End Analógico (AFE) de ECG para proporcionar un rendimiento de PPG y ECG altamente preciso y de bajo consumo.

El integrado MAX86150 funciona con una tensión de alimentación de $1,8 \mathrm{~V}$ con una fuente de alimentación independiente para los LED internos $(3,3 \mathrm{~V})$. La comunicación hacia y desde el módulo se realiza completamente a través de una interfaz estándar compatible con I2C. La comunicación I2C es un protocolo síncrono cuya principal característica es que utiliza dos líneas para transmitir la información: una para los datos (SDA) y otra para la señal de reloj (SCL). Cabe destacar que ambas líneas necesitan 
resistencias de pull-up. En la figura 2 se puede observar el circuito impreso del módulo MAX86150, destacando el diminuto tamaño de éste.

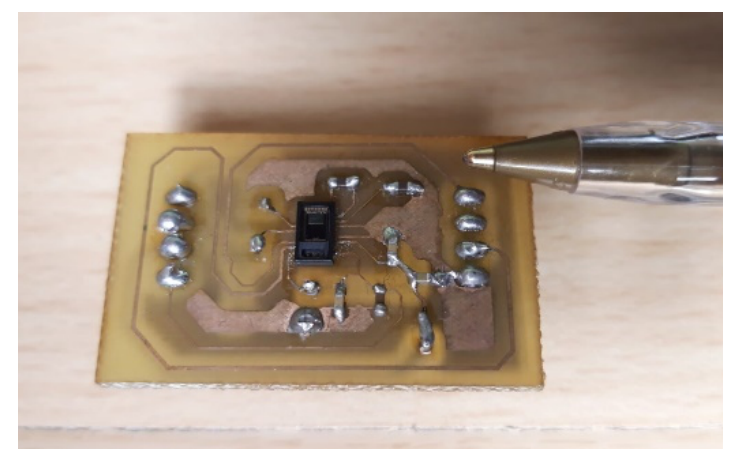

Figura 2: Módulo MAX86150

Dicho módulo es capaz de llevar a cabo una buena adquisición de la señal de electrocardiograma solamente con dos electrodos debido a que el amplificador de instrumentación que contiene el integrado MAX86150 posee un elevado factor de rechazo al modo común (CMRR). A la entrada de cada uno de los electrodos (positivo y negativo) se ha implementado un filtro R-C $(\mathrm{H}(\mathrm{w}))$ de paso bajo con el objetivo de eliminar las componentes de alta frecuencia asociadas a ruido. Para ello se ha usado una resistencia (R) de un valor de $50 \mathrm{k} \Omega$ y un condensador (C) de $10 \mathrm{nF}$, dando como resultado una frecuencia de corte (fc) de $318,31 \mathrm{~Hz}$ tal y como se muestra en las siguientes fórmulas (1) (2). Dicha frecuencia es mayor al espectro frecuencial de la señal ECG, que va desde los $0 \mathrm{~Hz}$ a los $100 \mathrm{~Hz}$ [16].

$$
H(w)=\frac{1}{1+j w \cdot R \cdot C}=\frac{1}{1+j w \cdot 50 \cdot 10^{3} \cdot 10 \cdot 10^{-9}}
$$

$$
f c=\frac{1}{2 \cdot \pi \cdot R \cdot C}=\frac{1}{2 \cdot \pi \cdot 50 \cdot 10^{3} \cdot 10 \cdot 10^{-9}}=318,31 \mathrm{~Hz}
$$

\subsection{SISTEMA DE MONITORIZACIÓN}

Como se ha comentado anteriormente, el sistema de monitorización de crisis epilépticas se ha diseñado mediante la combinación de una placa OpenBCI (EEG) y el módulo MAX86150 (ECG y PPG). Dado que el microcontrolador PIC32 que lleva incorporado la placa OpenBCI se comunica mediante el protocolo SPI y el integrado MAX86150 solo se puede comunicar mediante el protocolo $\mathrm{I} 2 \mathrm{C}$, no se pueden comunicar de forma directa. Por lo que se optó por llevar a cabo la comunicación entre ambos por medio de un microcontrolador Atmega328p. De tal forma que el microcontrolador Atmega328p actúa como maestro y se comunica mediante el protocolo $\mathrm{I} 2 \mathrm{C}$ con el módulo MAX86150 y a su vez el microcontrolador Atmega328p actúa como esclavo (enviando la información leída por el módulo MAX86150 al PIC32) y se comunica mediante el protocolo SPI con el microcontrolador PIC32 de la placa de OpenBCI siendo este último maestro. De esta forma, programando adecuadamente dichos microcontroladores existe una sincronización entre ambos módulos que hace que se pueda almacenar la información leída por los sensores en la tarjeta SD de la placa OpenBCI para su posterior análisis. La frecuencia de muestreo de las señales bioeléctricas que se están monitorizando (EEG, ECG, PPG) está fijada en $250 \mathrm{~Hz}$. Cabe destacar que se ha añadido un botón (external trigger) que está conectado a una de las entradas digitales del PIC32 de tal forma que cuando dicho botón es pulsado es capaz de registrar y almacenar en la tarjeta SD eventos externos tales como crisis, cambio de actividades que pueden desencadenar una crisis, etc. De esta forma es más fácil diferenciar y separar los períodos de tiempo de los pacientes y relacionar las variables bioeléctricas almacenadas con las actividades que realiza el paciente. En la figura 3 se puede observar el esquema del funcionamiento y comunicación del sistema de monitorización diseñado.

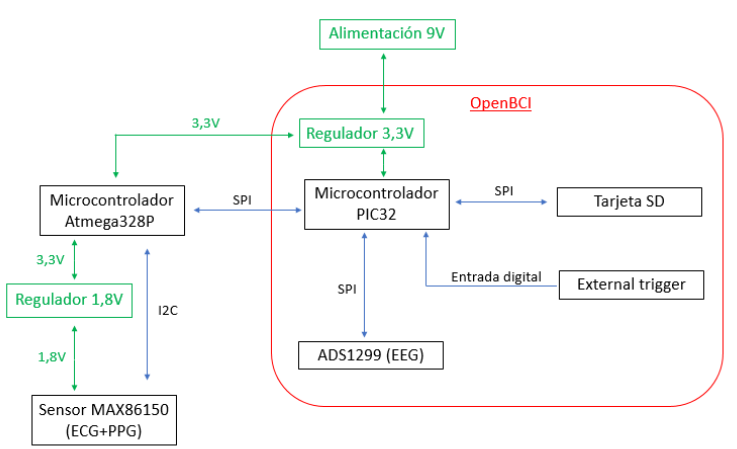

Figura 3: Esquema del sistema de montorización

Respecto a la alimentación del sistema, se lleva a cabo mediante una pila de $9 \mathrm{~V}$ directamente conectada la placa OpenBCI, la cual lleva incorporada un regulador de 3,3V que alimenta a su microcontrolador PIC32 y a sus respectivos integrados y módulos. A través del citado regulador se alimenta también al microcontrolador Atmega328P, el cual a su vez alimenta al integrado MAX86150 y a otro regulador de $1,8 \mathrm{~V}$ que se utiliza también para alimentar al integrado MAX86150 (ya que necesita 2 niveles de alimentación para poder obtener ECG y PPG de forma simultánea).

El microcontrolador PIC32 almacena los datos en formato hexadecimal en la tarjeta SD por bloques, es decir, a medida que recibe el microcontrolador los 
datos de los sensores, y cuando un bloque está completo (512 bytes) se escriben dichos datos en el archivo de la tarjeta SD. De esta manera se lleva a cabo una escritura eficiente de los datos en la tarjeta SD.

Con el fin de que quedase todo el sistema compacto y fuera portátil, se ha diseñado una carcasa que alberga parte de la electrónica anteriormente citada. De tal forma que la placa OpenBCI está fijada a la carcasa mediante tornillos y justo debajo de la placa se encuentra la alimentación del sistema (pila de 9V) y el microcontrolador Atmega328p. Como se puede observar en la figura 4 del microcontrolador Atmega328p sale un cable negro encargado de comunicarse con el módulo MAX86150.

Para el módulo MAX86150 también se diseñó una carcasa de color blanco en la cual se apoya el dedo y se fija el dedo con velcro para medir PPG. De ella sale el cable negro descrito en el párrafo anterior y dos electrodos que son colocados en el pecho con el objetivo de medir ECG. Los electrodos que se han utilizado son electrodos desechables pre-gelificados con contenido de cloruro de plata.

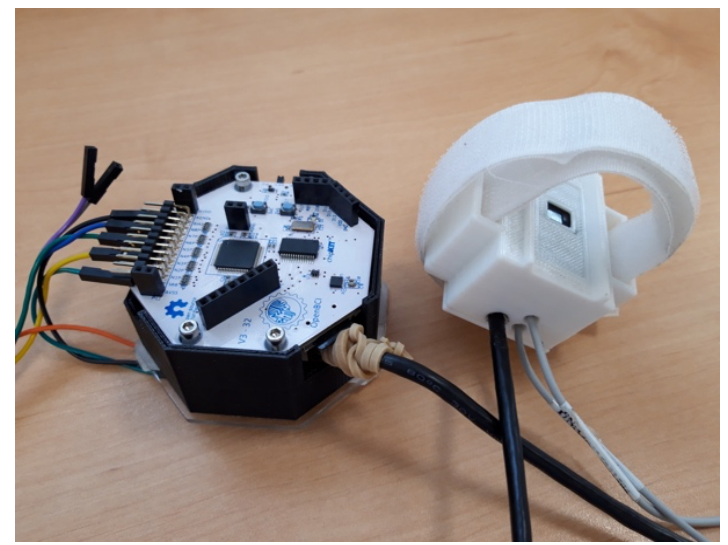

Figura 4: Carcasas

Para llevar a cabo la adquisición de la señal de EEG de oído, se ha diseñado un soporte con la forma adecuada para ser colocado detrás de la oreja derecha (lóbulo temporal). Dicho soporte ha sido fabricado con goma EVA (etilvinilacetato) y tiene incorporados los soportes en los que se insertan los 4 electrodos de copa de oro ( 3 canales +1 referencia). Para llevar a cabo la sujeción del soporte de goma EVA diseñado se han utilizado dos turbantes. De esta manera, el soporte se queda fijo haciendo presión los electrodos sobre la piel de los pacientes, obteniéndose así una buena calidad en las adquisiciones de la señal de EEG. Los electrodos de copa de oro que se han usado en la experimentación son reutilizables y están diseñados para dar señales fiables con facilidad de uso una y otra vez. En la figura 5 se puede visualizar el sistema completo de monitorización, incluida la Tablet que se comunica con el sistema mediante un dongle de bluetooth solamente para gestionar el tiempo de grabación del sistema de monitorización.

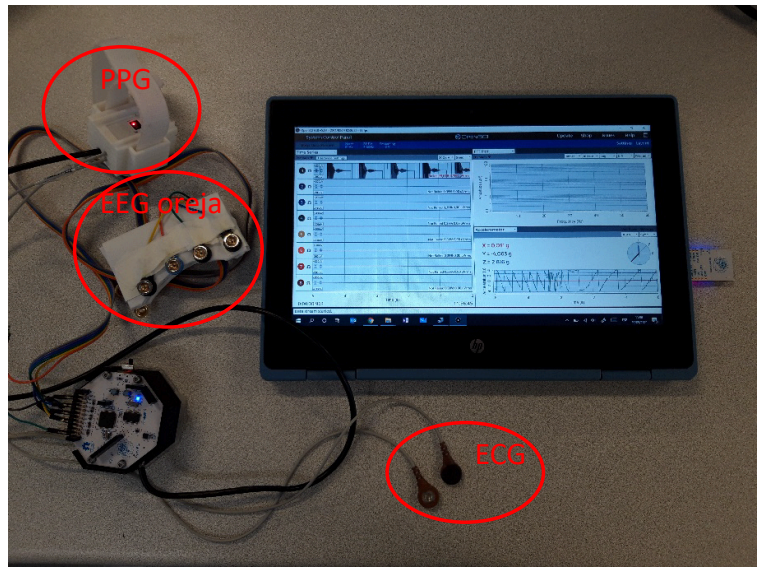

Figura 5: Sistema de monitorización

\subsection{EXPERIMENTACIÓN}

Para llevar a cabo la validación de los datos adquiridos por el sistema de monitorización se realizaron grabaciones en 5 voluntarios (tabla 1), con una duración total de 20 minutos cada grabación.

Tabla 1: Información de los voluntarios.

\begin{tabular}{|c|c|c|}
\hline Género & Edad (años) & Peso (Kg) \\
\hline Masculino & 26 & 78 \\
Masculino & 28 & 70 \\
Femenino & 24 & 63 \\
Masculino & 47 & 72 \\
Femenino & 23 & 65 \\
\hline
\end{tabular}

Durante los 15 primeros minutos el sujeto debía permanecer con los ojos cerrados, posteriormente debía llevar a cabo 1 minuto de apertura y cierre de ojos. Seguidamente, durante 3 minutos debía de hiperventilar (15-20 respiraciones/min). Finalmente, al voluntario se le sometía a 1 minuto de estimulación luminosa (parpadeo 2,5 Hz). Este procedimiento se diseñó en base a los consejos de una especialista en neurología cuyo trabajo se realiza en una unidad de videovigilancia para epilepsia. 


\subsection{TRATAMIENTO DE LOS DATOS}

Como se ha comentado anteriormente los datos son almacenados en la tarjeta SD en formato hexadecimal en un archivo de texto. Una vez que dichos datos se han convertido en valores decimales con signo, se ha procedido a llevar a cabo el análisis de estos mediante el software de Matlab. Para ello primero se ha realizado el filtrado de dichas señales, para la señal de ECG se ha utilizado un filtro paso banda de $0,4 \mathrm{~Hz}$ a $5 \mathrm{~Hz}$ y para la señal de PPG se utilizado un filtro paso banda de $0,4 \mathrm{~Hz}$ a $3 \mathrm{~Hz}$. En la figura 6 se puede observar en color rojo la señal de ECG en bruto y en color azul la señal ya filtrada. También se puede visualizar en la figura 7 la señal de PPG en bruto en color rojo y en color azul la señal ya filtrada.

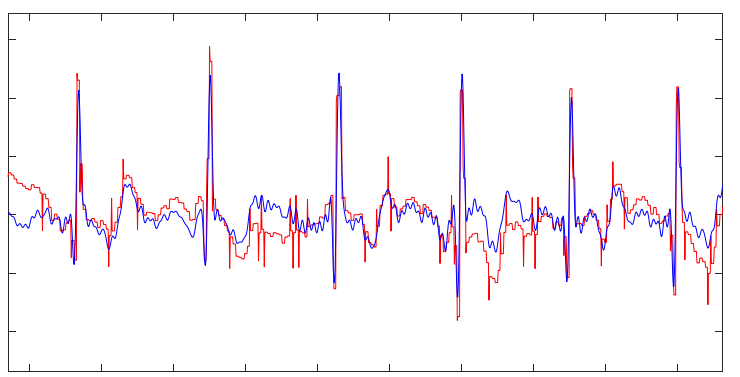

Figura 6: Señal ECG

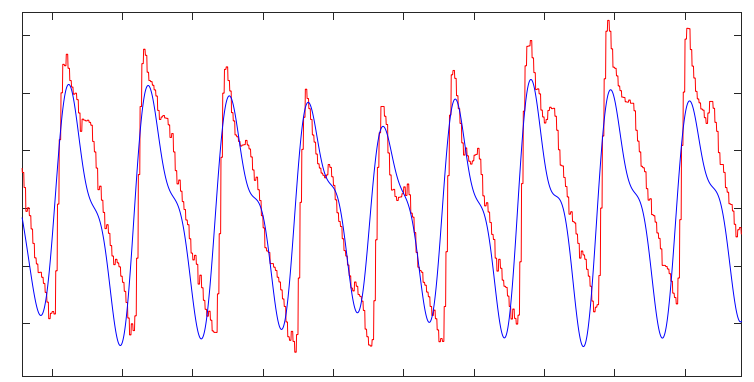

Figura 7: Señal PPG

Para cada una de las 3 señales de EEG de oído obtenidas, primero se utiliza un filtro de ranura a 50 $\mathrm{Hz}$ (para eliminar cualquier interferencia eléctrica) seguidamente dicha señal se filtra mediante un filtro de paso banda de $0,5 \mathrm{~Hz}$ a $80 \mathrm{~Hz}$. A continuación, se vuelve a filtrar otra vez la señal EEG mediante filtros de paso banda para obtener información de las diferentes ondas que la componen [10]:

- Ondas delta $(0,5-3,5 \mathrm{~Hz})$ : son las que tienen una mayor amplitud de onda y se relacionan con el sueño profundo.

- Ondas theta $(4-7,5 \mathrm{~Hz})$ : Se relaciona con las tareas cognitivas internas, capacidades imaginativas, con la reflexión y el sueño.
- Ondas alfa (8-13Hz): predomina cuando el Sistema Nervioso Central se encuentra en reposo, relajado pero despierto y atento.

- Ondas beta (13-30 Hz): Están asociadas a tareas cognitivas externas, actividades relacionadas con la concentración como puede ser resolver un problema matemático.

En la figura 8 se pueden observar las diferentes ondas que componen las señales de EEG obtenidas mediante el sistema propuesto en este artículo.

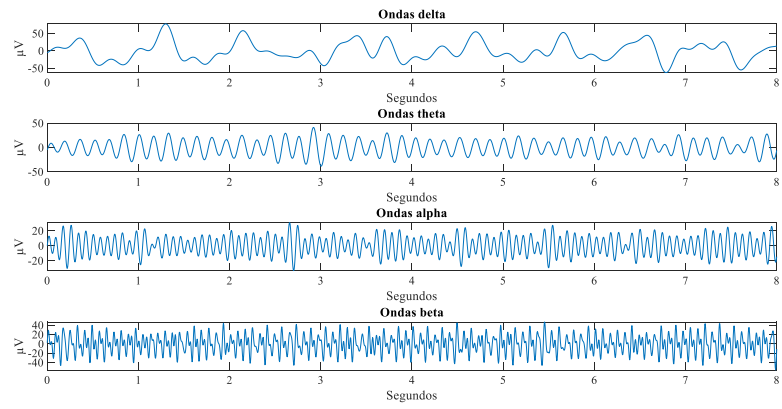

Figura 8: Ondas EEG.

\subsection{CÁLCULO DE PARÁMETROS}

Una vez se ha llevado a cabo el filtrado de las señales, se ha procedido a calcular la variabilidad del ritmo cardiaco (HRV) y el tiempo de tránsito de pulso (PTT) a partir de la señal de ECG y PTT. Tal y como se muestra en la figura 9, el parámetro HRV se obtiene a partir de la señal de ECG calculando el tiempo entre los picos R. Para llevar a cabo el cálculo del parámetro PTT es necesario medir el tiempo que transcurre entre el pico R de la señal de ECG y el valor máximo que se alcanza posteriormente en la señal de PPG. Ambos parámetros son calculados en milisegundos. En la figura 9 se puede visualizar la señal de ECG (en azul) y PPG (en rojo), y como se lleva a cabo el cálculo de los parámetros HRV y PTT.

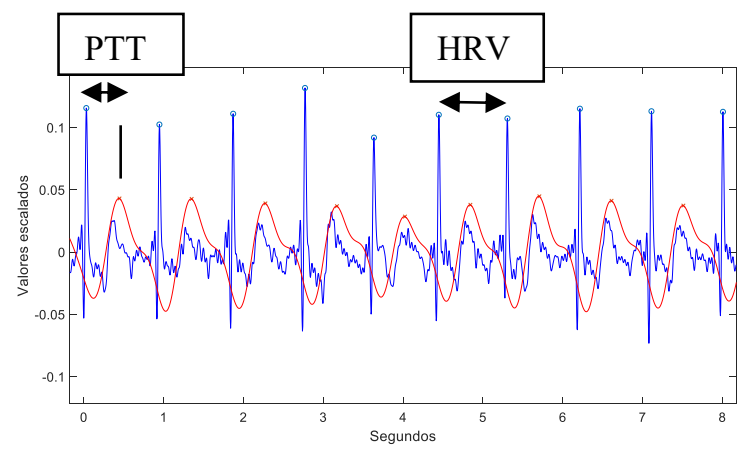

Figura 9: Señal ECG y PPG. 


\section{RESULTADOS}

En este apartado se va a realizar un análisis de los resultados obtenidos, y las distintas líneas futuras de investigación.

\subsection{CONCLUSIONES}

Como se ha ido desarrollando en el epígrafe anterior, se ha logrado con éxito desarrollar un sistema portátil de monitorización de crisis epilépticas. Cabe destacar la elevada calidad de las señales obtenidas mediante los sensores que se han descrito en el artículo. Gracias a dicha calidad es posible calcular de manera exacta las diferentes bandas que componen el EEG y los parámetros HRV y PTT.

$\mathrm{Si}$ analizamos los datos obtenidos tras la experimentación que se ha propuesto anteriormente, podemos observar en la figura 10 cómo al hiperventilar (min 16-19) se produce un descenso tanto en los valores del parámetro HRV Y PTT. Este fenómeno se ha observado en los 5 casos estudiados.

Ese descenso se traduce en un aumento del ritmo cardíaco y un aumento de la presión arterial, ya que la hiperventilación provoca un aumento de oxígeno y disminución en la cantidad de dióxido de carbono en la sangre. De manera que se libera menos oxígeno a los tejidos, provocando un aumento en la potencia y frecuencia de los latidos del corazón [17].
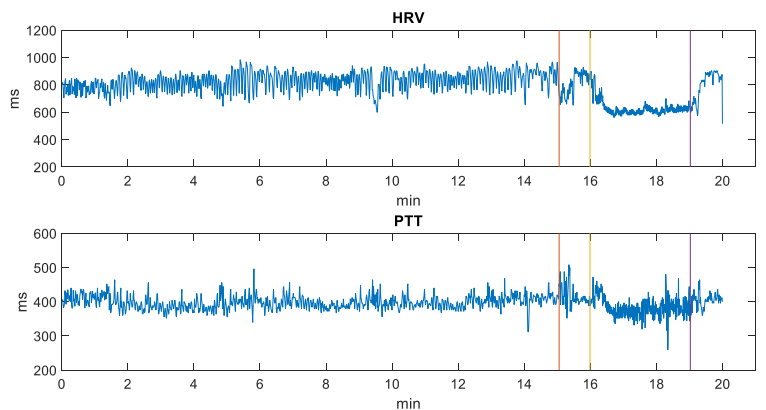

Figura 10: Variación parámetros HRV y PTT

Como conclusión, el dispositivo propuesto permite obtener todas las señales descritas anteriormente (ECG, PPG, EEG) de forma ambulatoria con un nivel de calidad similar al que se obtiene con los sistemas estáticos usados en las unidades de videovigilancia de los hospitales. Por lo tanto, dicho dispositivo es capaz de aportar valiosa información acerca del sistema nervioso y cardiovascular con el objetivo de estudiar de manera ambulatoria las crisis epilépticas.

\subsection{LÍNEAS FUTURAS}

El dispositivo que se presenta en este documento puede servir como instrumento de apoyo a la hora de tomar decisiones clínicas. Por ello se espera seguir avanzando en este campo y que el dispositivo descrito sirva de base para poder llegar a detectar o predecir los inicios de las crisis lo antes posible, a partir de la información proporcionada por los sensores mediante técnicas de inteligencia artificial.

\section{Agradecimientos}

Los autores quieren agradecer el apoyo económico a la Agencia Estatal de Investigación (AEI) mediante el proyecto PID2019-111023RB-C32 AEI/10.13039/501100011033. David ZambranaVinaroz agradece el apoyo económico del programa ACIF con la beca ACIF/2019/058.

\section{English summary}

\section{AMBULATORY EPILEPTIC SEIZURE MONITORING SYSTEM}

\begin{abstract}
Epilepsy is a chronic disease with an important social impact. Those affected and their families often live conditioned by the possibility of an epileptic seizure and its possible consequences such as accidents, injuries and even sudden unexplained death (SUDEP). Therefore, this article proposes the design of an electronic and portable device that integrates and stores the information collected by different sensors (EEG, ECG and PPG) in order to calculate certain parameters that provide information about the nervous and cardiovascular system. In addition, such a device can be used to study epileptic seizures on an outpatient basis and serve as a clinical decision support tool.
\end{abstract}

Keywords: Epilepsy, portable, monitoring, ECG, EEG, PPG, PTT.

\section{Referencias}

[1] Baulac M, de Boer H, Elger C, Glynn M, Kälviäinen R, Little A, Mifsud J, Perucca E, Pitkänen A, Ryvlin P. (2015) "Epilepsy priorities in Europe: A report of the ILAE-IBE Epilepsy Advocacy Europe Task Force". Epilepsia. pp.1687-95. 
[2] Begley CE, Durgin TL. (2015) "The direct cost of epilepsy in the United States: A systematic review of estimates". Epilepsia. pp.1376-87.

[3] Block, R.C., Yavarimanesh, M., Natarajan, K. et al. Conventional pulse transit times as markers of blood pressure changes in humans. Sci Rep.

[4] DeGiorgio CM, Miller P, Meymandi S, et al. (2010) "RMSSD, a measure of vagus-mediated heart rate variability, is associated with risk factors for SUDEP: the SUDEP-7 Inventory" Epilepsy Behav. pp.78-81.

[5] Furrukh Sana, Eric M. Isselbacher MD, Jagmeet P. Singh, (2020) "Wearable Devices for Ambulatory Cardiac Monitoring" JACC Journal of the American College of Cardiology, pp.1582-1592.

[6] Jehi L, Schuele S. (2015), "Sudden death in epilepsy: Where is the "heart" of the problem?" Neurology. pp.208-9.

[7] Hoja de características del módulo MAX86150; https://datasheets.maximintegrated.com/en/ds/ $\underline{\text { MAX86150.pdf }}$

[8] OpenBCI; www.openbci.com

[9] Pedro J. Serrano-Castro, Jose Angel MauriLlerda, Francisco José Hernández-Ramos, et al, Beatriz Parejo-Carbonell, Pablo QuirogaSubirana, Fernando Vázquez-Gutierrez, Sonia Santos-Lasaosa, Carolina Mendez-Lucena, Luis Redondo-Verge, Carlos Tejero-Juste, Clara Morandeira-Rivas, Jerónimo Sancho-Rieger, Jorge Matías-Guiu. (2015) "Adult Prevalence of Epilepsy in Spain: EPIBERIA, a PopulationBased Study", The Scientific World Journal.

[10] Picon Calderon, Cristian; Gomez Tinoco, Saul. (2017). Electrofisiología neuronal: Ritmos cerebrales y epilepsia.

[11] R. García-Ramos, A. García Pastor, J. Masjuan, C. Sánchez, A. Gil, (2011) "FEEN: Informe sociosantario FEEN sobre la epilepsia en España”, Neurología, pp.548-555.
[12] Rosales-Soto, G.; Corsini-Pino, R.; MonsalvesAlvarez, M. ; Yanez-Sepulveda, R. (2021) "Respuesta del balance simpático-parasimpático de la variabilidad de la frecuencia cardíaca durante una semana de entrenamiento aeróbico en ciclistas de ruta". Rev Andal Med, pp.143147.

[13] Ryvlin P, Nashef L, Lhatoo SD, Bateman LM, Bird J, Bleasel A, Boon P, Crespel A, Dworetzky BA, Høgenhaven $\mathrm{H}$, Lerche $\mathrm{H}$, Maillard L, Malter MP, Marchal C, Murthy JM, Nitsche M, Pataraia E, Rabben T, Rheims S, Sadzot B, Schulze-Bonhage A, Seyal M, So EL, Spitz M, Szucs A, Tan M, Tao JX, Tomson T. (2013) "Incidence and mechanisms of cardiorespiratory arrests in epilepsy monitoring units (MORTEMUS): a retrospective study" Lancet Neurol.pp.966-77.

[14] Sana F, Isselbacher EM, Singh JP, Heist EK, Pathik B, Armoundas AA.(2020), "Wearable Devices for Ambulatory Cardiac Monitoring: JACC State-of-the-Art Review" J Am Coll Cardiol. pp.1582-1592.

[15] Serhani MA, T El Kassabi H, Ismail H, Nujum Navaz A. (2020), "ECG Monitoring Systems: Review, Architecture, Processes, and Key Challenges" Sensors (Basel). pp.1796.

[16] Vidal-SilvaC., \& Gatica-RojasV. (2013). Diseño e implementación de un sistema electrocardiográfico digital. Revista Facultad De Ingeniería Universidad De Antioquia, pp.99-107

[17] Wood, Cristina ; Cano Vindel, Antonio. (2021) "La Hiperventilación y el Trastorno de Angustia a la Luz de un Marco Cognitivo". Clínica y Salud . pp.57-66.

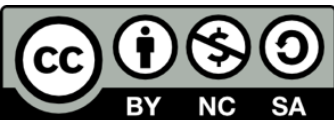
(C) 2021 by the authors. Submitted for possible open access publication under the terms and conditions of the Creative Commons Attribution CC BY-NC-SA 4.0 license (https://creativecommons.org/licenses/byncsa/4.0/deed.es). 